\title{
Hypothalamic-pituitary-adrenal axis activity in patients with primary polydipsia compared to healthy volunteers
}

Clara Odilia Sailer ( $\square$ cosailer@mgh.harvard.edu )

Universitatsspital Basel https://orcid.org/0000-0001-6439-8191

Jill M. Kühne

Universitätsspital Basel: Universitatsspital Basel

Ismael da Conceição

Universitätsspital Basel: Universitatsspital Basel

Julie Refardt

Universitätsspital Basel: Universitatsspital Basel

Mirjam Christ-Crain

Universitätsspital Basel: Universitatsspital Basel

Bettina Winzeler

Universitätsspital Basel: Universitatsspital Basel

\section{Research Article}

Keywords: Stress axis, stress regulation, salivary cortisol, 24-hour urinary cortisol, compulsive fluid intake

Posted Date: March 3rd, 2022

DOI: https://doi.org/10.21203/rs.3.rs-1370576/v1

License: (c) (i) This work is licensed under a Creative Commons Attribution 4.0 International License.

Read Full License 


\section{Abstract}

Objective: Primary polydipsia is characterized by excessive fluid intake which may suppress vasopressin levels. It is speculated that suppressed vasopressin levels lead to a dysregulated hypothalamic-pituitaryadrenal (HPA) axis as vasopressin co-modulates the HPA axis. However, data is contradictory. The aim of this study was to investigate markers of the HPA axis in patients with primary polydipsia compared to healthy controls.

Design: Exploratory analysis combining data from two different prospective observational studies.

Method: We included 34 patients with primary polydipsia (68\% females, median aged 29.5 years (IQR 26.0, 38.8)) and 20 healthy volunteers (55\% females, median age 24.0 years (IQR 22.0, 27.2)). The main outcome was circadian serum and salivary cortisol, 24-hour urinary free cortisol, and cortisol levels before and after adrenocorticotropic hormone (ACTH) stimulation.

Results: No difference was seen in circadian serum cortisol levels $(p=0.9)$, urinary free cortisol levels $(p=0.17)$, and serum cortisol in response to ACTH stimulation $(p=0.77)$ between patients with primary polydipsia and healthy volunteers. However, circadian salivary cortisol levels were significantly lower in patients with primary polydipsia as compared to healthy volunteers with an estimated difference of -3.7 $n m o l / l(95 \%-C l-5.5,-1.8 \mathrm{nmol} / \mathrm{l}, \mathrm{p}=<0.001)$.

Conclusion: Our results suggest no difference in HPA axis activity between patients with primary polydipsia and healthy volunteers. The observed difference in salivary cortisol levels may be linked to a dilution effect in saliva rather than an altered stress axis considering the other findings.

\section{Introduction}

Primary polydipsia is characterized by increased thirst sensation, excessive fluid intake, and secondary polyuria (24h urinary output $>50 \mathrm{ml} / \mathrm{kg}$ body weight) [1-3]. Although vasopressin, the main regulatory hormone in fluid homeostasis, has been shown to be involved in stress regulation, the interplay of primary polydipsia and the hypothalamic pituitary adrenal (HPA) axis is not well understood. It is speculated that the constantly increased fluid intake leads to suppressed vasopressin levels and thus a downregulation of the HPA axis [4]. Since primary polydipsia is often associated with schizophrenia spectrum disorders [3-5], most research on stress adaptivity and HPA axis activity has been done in patients with primary polydipsia and chronic schizophrenia [6-11]. Goldman et al. have shown that patients suffering from schizophrenia with primary polydipsia have a different stress response than patients suffering from schizophrenia without primary polydipsia [7, 9]. More precisely, in patients with schizophrenia and primary polydipsia, Goldman et al. observed more often non-suppression of cortisol following a dexamethasone suppression test, than in patients without primary polydipsia [9]. Similarly, serum cortisol in response to ACTH stimulation was found to be enhanced, albeit marginally, in polydiptic patients with schizophrenia compared to healthy controls [7]. These results suggest that in patients with primary polydipsia and schizophrenia the HPA axis activity seems to be upregulated $[7,10,11]$. 
Recent studies have shown an increasing number of patients with habitual polydipsia, i.e., without associated psychiatric disorder [3,12,13], mainly in health conscious individuals. Whether primary polydipsia in these patients is also linked to an altered HPA axis activity is unknown.

The aim of this study was therefore to investigate the HPA axis activity, i.e., circadian serum and salivary cortisol levels, 24-hour urinary cortisol, and cortisol in response to adrenocorticotropic hormone (ACTH) stimulation, in a larger collective of patients with psychogenic and habitual primary polydipsia and to compare the results to healthy volunteers.

\section{Methods}

\section{Study design and participants}

This study combined data from two prospective studies conducted at the University Hospital Basel, Switzerland. The methodology of both studies was similar with the aim to elucidate the effect of GLP-1 receptor agonists on drinking behavior in 34 patients with primary polydipsia [14] and in 20 healthy volunteers [15] in a randomized placebo-controlled crossover design. For the present exploratory analysis, only data of participants during the placebo phase were evaluated. Full details of the studies' rationale, design, and statistical analysis have been published elsewhere [14, 15].

Inclusion criteria for patients with primary polydipsia were age $\geq 18$ years, polyuria of $>50 \mathrm{ml} / \mathrm{kg} / \mathrm{day}$, and polydipsia of $>3000 \mathrm{ml} /$ day. Exclusion criteria included secondary polydipsia such as central or nephrogenic diabetes insipidus, diabetes mellitus, hypokalemia, or hypercalcemia, history of pancreatitis, severe renal insufficiency, and cancer.

Eligible participants for healthy volunteers were age $\geq 18$ years and $\leq 65$ years without regular drug intake, except for oral contraceptives and no history of primary or secondary polydipsia.

Pregnancy and breastfeeding were exclusion criteria in both studies.

\section{Study objective and outcomes}

The objective of the present study was to explore differences in HPA axis activity in patients with primary polydipsia compared to healthy volunteers. The HPA axis activity was assessed using the following outcome measures: circadian serum and salivary cortisol levels, 24-h free urinary cortisol levels and serum cortisol levels in response to ACTH stimulation.

\section{Study procedure}

Patients with primary polydipsia and healthy volunteers arrived at the study site at 8:00 a.m. following an overnight fast (no intake of any food and fluids, including water) of 12 hours and were observed until 4:00 p.m. of the same day. Standardized meals were served at fixed times and participants had unlimited access to water but were blinded to the consumed amount of water. Water intake was recorded between 8.00 a.m. and 4:00 p.m. 
Circadian serum and salivary cortisol levels: At patients' arrival, a venous catheter was placed into an antecubital vein. Blood for serum cortisol levels was drawn at 8:00 am, 12:00 pm, and 4:00 pm. Saliva for salivary cortisol levels was collected at 8:00 am, 12:00 pm, and 4:00 pm. Before leaving the study center, participants were instructed to collect saliva at 8:00 pm and 12:00 am of the same day for later cortisol level analysis. Participants were instructed not to eat, chew gum, or brush their teeth 30 minutes prior to saliva collection.

24-h free urinary cortisol levels: Urine collection was started at 8:00 am for the measurement of 24-h free urinary cortisol levels. Before leaving the study center at 4:00 pm, participants were instructed to collect their urine until 8:00 am of the next day for later urinary cortisol analysis. Free urinary cortisol was computed by measuring the urinary cortisol per liter and multiplied with the voided volume over 24 hours $(=\mathrm{nmol} / 24-\mathrm{h})$.

ACTH stimulation test: On the second day, participants presented at the study center at 8:00 am and a venous catheter was placed into an antecubital vein. After basal blood sampling, $1 \mu \mathrm{g} \mathrm{ACTH}$ (Synacthen ${ }^{\circledR}$ ) was injected and a second blood sampling took place after 25 minutes, both for the analysis of serum cortisol levels.

\section{Laboratory measurements}

Blood samples for serum cortisol levels were processed as routine laboratory measurements in the central laboratory of the hospital. All serum, urinary, and salivary cortisol levels were determined with the Elecsys Cortisol Test 2010 (Roche Diagnostics GmbH, Mannheim, Germany), an

electrochemiluminescence immunoassay with a limit of detection between 0.5 and $1750 \mathrm{nmol} / \mathrm{l}$. Further laboratory parameters (e.g., plasma sodium, plasma potassium, plasma chloride) were processed as routine laboratory measurements in the central laboratory of the hospital.

\section{Statistical analysis}

This is a predefined secondary analysis combining data from two different studies with the same study methodology. The primary objective of this analysis was to identify a difference in the HPA axis activity in patients with primary polydipsia compared to healthy volunteers. Baseline characteristics are shown as median with interquartile range (IQR), and number (n) with percentage (\%), respectively. The Fisher's Exact Test was used to test two-group differences of categorical variables and the Wilcoxon Rank Sum Test was used to compare two-group differences of continuous variables. Mixed effect models were used for daily changes in serum and salivary cortisol with timepoint as fixed effect and patients as random effect. As auxiliary analysis, we included sex, type of polydipsia (patients with psychiatric comorbidities were categorized as "psychogenic polydipsia" and those without psychiatric comorbidities as "habitual polydipsia") and severity of polydipsia (in sight of a lacking official threshold, we categorized patients with a fluid intake below the median overall fluid intake of $4500 \mathrm{ml}$ as "moderate polydipsia" whereas patients with a fluid intake equal to or above $4500 \mathrm{ml}$ as "severe polydipsia") as random effect. The Spearman's Rank Correlation Coefficient was computed to assess correlation between 24h-h drinking amount and 24-h urinary amount with free urinary cortisol. Delta serum cortisol levels in response to 
ACTH stimulation was computed by subtracting stimulated minus basal serum cortisol levels. Serum cortisol levels in response to ACTH stimulation were compared between patients with primary polydipsia and healthy volunteers using a mixed effects linear model including timepoint, i.e., basal and stimulated cortisol levels, as fixed effect and patients as random effects. Subgroup analysis included type and severity of polydipsia. The model was adjusted for age, sex, and intake of oral contraceptive pill.

For all analyses, graphical representation was done using boxplots indicating the median and interquartile range in the box and the whiskers including 2.5 times of the IQR.

Statistical analyses were performed using the statistic program R Statistical Software[16]. Hypothesis testing was two-sided and $p$-values $<0.05$ were considered statistically significant.

\section{Results}

\section{Baseline Characteristics:}

Primary polydipsia: 34 patients with primary polydipsia (68\% female) aged 29.5 years (IQR 26.0, 38.8) with a median body mass index (BMI) of $23.1 \mathrm{~kg} / \mathrm{m}^{2}$ (IQR 20.7, 25.5) were included in the analysis. The median reported fluid intake was $4500 \mathrm{ml} /$ day (IQR 4000, 5000). 17 patients were subclassified as moderate polydipsic (median reported fluid intake $4000 \mathrm{ml} /$ day [IQR 4000, 4500]) and 17 patients were classified as severe polydipsic (median reported fluid intake was $5500 \mathrm{ml} /$ day [IQR 5000, 6000]). 20 (59\%) patients were classified as having habitual polydipsia and $14(41 \%)$ as psychogenic polydipsia, based on psychiatric comorbidities, including depression $(n=10)$, schizophrenia $(n=1)$, anxiety disorder $(n=1)$, borderline personality disorder $(n=1)$ and sleep disorder $(n=1)$. Four $(16 \%)$ of the twenty-five female participants took oral contraceptive pills during the time of the study. All routine laboratory parameters were in the normal range (Table 1). 
Table 1

Baseline characteristics of patients with primary polydipsia and healthy volunteers

Primary polydipsia

34

Number of participants

Baseline Characteristics

Female sex (\%)

Age (years)

Caucasian

BMI $\left(\mathrm{kg} / \mathrm{m}^{2}\right)$

Systolic blood pressure $(\mathrm{mmHg})$

Diastolic blood pressure $(\mathrm{mmHg})$

Heart rate (bpm)

Current smoker

Alcohol consumption (glass/week)

Drinking habits

Amount of drinking ( $\mathrm{ml} /$ day)

Severity of polydipsia

Moderate polydipsia (ml/day)

Severe polydipsia (ml/day)

Comorbidities

Psychiatric comorbidity

Psychogenic polydipsia

Depression

Schizophrenia

Anxiety disorder

Others

Medication
$9(26)$

1 (3)

1 (3)

2 (6)
Healthy volunteers

p-value
23 (68)

$29.5[26.0,38.8]$

$34(100)$

$23.1[20.7,25.5]$

$121.5[112.0,139.2]$

$75.0[68.2,79.8]$

$74.0[66.2,85.8]$

11 (32)

$1.0[0.0,3.0]$

$4500[4000,5000]$

$2000[2000,2313]$

$<0.001$

$11(55)$

$24.0[22.0,27.2]$

20 (100)

$23.0[21.1,23.8]$

$127[115.2,133.2]$

$80.5[70.8,85.2]$

$66.0[60.8,78.0]$

9 (47)

0.432

$3.0[2.0,4.2]$

0.036

4000 [4000, 4500]

$5500[5000,6000]$

$14(41)$

$0(0)$

0.003

Continuous variables are presented as median (IQR); categorical variables are presented as frequency (percentage). Two-group comparison was computed using the Wilcoxon Rank Sum Test for continuous variables and the Fishers Exact test for categorical variables, respectively. 


\begin{tabular}{|c|c|c|c|}
\hline & Primary polydipsia & Healthy volunteers & p-value \\
\hline Oral contraceptive pill & $4(12)$ & $6(30)$ & 0.193 \\
\hline Antidepressants & $7(21)$ & $0(0)$ & 0.079 \\
\hline \multicolumn{4}{|l|}{ Laboratory parameters } \\
\hline Plasma sodium (mmol/l) & $140.0[139.0,141.8]$ & $141.0[139.0,141.0]$ & 0.691 \\
\hline Plasma potassium (mmol/l) & $3.8[3.7,4.0]$ & $3.8[3.7,4.0]$ & 0.416 \\
\hline Plasma chloride (mmol/l) & $103.0[102.0,105.0]$ & $103.0[102.0,104.0]$ & 0.584 \\
\hline Plasma creatinine (mmol/l) & $69.5[61.2,81.2]$ & $73.0[70.0,82.5]$ & 0.210 \\
\hline GFR (ml/min/1.73m) & $104.0[96.0,116.0]$ & $105.0[96.5,118.0]$ & 0.761 \\
\hline Plasma glucose $(\mathrm{mmol} / \mathrm{l})$ & $4.8[4.4,5.2]$ & $4.7[4.5,4.8]$ & 0.289 \\
\hline HbA1c (\%) & $5.0[4.7,5.1]$ & $4.9[4.7,5.1]$ & 0.881 \\
\hline Plasma osmolality (mosm/kg) & $289.0[286.0,292.0]$ & $289.0[282.0,291.0]$ & 0.302 \\
\hline $24 \mathrm{~h}$ urinary osmolality $(\mathrm{mosm} / \mathrm{kg})$ & $216.5[153.8,269.0]$ & $470.0[329.2,655.8]$ & $<0.001$ \\
\hline \multicolumn{4}{|c|}{$\begin{array}{l}\text { Continuous variables are presented as median (IQR); categorical variables are presented as frequency } \\
\text { (percentage). Two-group comparison was computed using the Wilcoxon Rank Sum Test for } \\
\text { continuous variables and the Fishers Exact test for categorical variables, respectively. }\end{array}$} \\
\hline
\end{tabular}


Table 2

HPA axis activity of Patients with Primary Polydipsia and Healthy Volunteers

\section{Primary polydipsia Healthy volunteers}

p-value

Number of participants

Serum circadian cortisol

08:00 am serum cortisol (nmol/l)

12:00 pm serum cortisol ( $\mathrm{nmol} / \mathrm{l})$

04:00 pm serum cortisol ( $\mathrm{nmol} / \mathrm{l})$

Salivary circadian cortisol

08:00 am salivary cortisol ( $\mathrm{nmol} / \mathrm{l})$

12:00 pm salivary cortisol ( $\mathrm{nmol} / \mathrm{l})$

04:00 pm salivary cortisol $(\mathrm{nmol} / \mathrm{l})$

08:00 pm salivary cortisol ( $\mathrm{nmol} / \mathrm{l})$

12:00 am salivary cortisol $(\mathrm{nmol} / \mathrm{l})$

Urinary cortisol

24h-urinary cortisol (nmol/24h)

ACTH test

Basal serum cortisol (nmol/l)

Stimulated serum cortisol (nmol/l)

Continuous variables are presented as median (IQR) and two-group comparison was computed using the Wilcoxon Rank Sum Test.
34

20

$423[345,540]$

$262[202,302]$

$211[166,271]$

$13.5[10.1,22.8]$

$24.1[16.2,31.8]$

0.039

$5.2[3.6,6.8]$

$8.5[5.5,13.9]$

0.001

$3.8[3.0,7.8]$

$8.5[7.4,12.1]$

$<0.001$

$3.0[3.0,5.6]$

$4.0[3.4,8.5]$

0.199

$4.2[3.0,8.3]$

$5.9[4.4,9.0]$

0.147
$154[100,234]$

$445[359,551]$

$618[580,684]$
$189[133,338]$

0.169

Healthy volunteers: The group of the healthy volunteers included 20 participants ( $55 \%$ female) aged 24.0 years (IQR 22.0, 27.2) with a median BMI of $22.9 \mathrm{~kg} / \mathrm{m}^{2}$ (IQR 21.1, 23.8). The median reported fluid intake was $2000 \mathrm{ml} /$ day (IQR 2000, 2300). None of the participants had psychiatric comorbidities. Six (55\%) of the eleven female participants took oral contraceptive pills during the time of the study. All routine laboratory parameters were in the normal range. (Table 1).

\section{HPA axis activity}

\section{Circadian serum and salivary cortisol levels}

The circadian serum cortisol levels were similar in patients with primary polydipsia compared to healthy volunteers ( $p$-value $=0.9)($ Fig. 1a). Stratified for sex in the whole sample, there was no difference in the circadian serum cortisol levels ( $p$-value 0.6 ) but female patients on oral contraceptive pills $(n=10)$ had 
significantly higher cortisol levels (estimated difference $184 \mathrm{nmol} / \mathrm{l}(95 \%-\mathrm{Cl} 125,243), \mathrm{p}$-value $<0.001)$ compared to females without oral contraceptives. In patients with primary polydipsia only, there was no difference in the circadian cortisol levels in the subgroup type of polydipsia (habitual vs. psychogenic polydipsia, $p$-value $=0.6$ ), nor in the subgroup severity of polydipsia (moderate vs. severe polydipsia, $p$ value $=0.8$.

We found a significant difference in circadian salivary cortisol levels. In a mixed effect model, the estimated difference was $-3.8 \mathrm{nmol} / \mathrm{l}(\mathrm{Cl}-5.5,-1.8 \mathrm{nmol} / \mathrm{l}$ for patients with primary polydipsia ( $\mathrm{p}$-value < 0.001) (Fig. 1b). Stratified for sex, the estimated difference between patients with primary polydipsia and healthy volunteers remained statistically significant ( $p$-value $<0.001)$. We found no difference in the subgroups of type of polydipsia (habitual vs. psychogenic polydipsia, $p$-value $=0.7$ ), nor for severity of polydipsia (moderate vs. severe polydipsia, $p$-value $=0.5$ ).

\section{4-hour urinary free cortisol levels}

There was no difference in 24-hour urinary free cortisol levels between patients with primary polydipsia compared to healthy volunteers $(p-v a l u e=0.44)(F i g .2)$. Furthermore, there was no correlation between drinking amount or urine amount and 24-hour urinary free cortisol $(R=-0.2$ for drinking amount over 8 h, and $\mathrm{R}=0.03$ for urine amount). There was no difference in type of polydipsia (habitual vs. psychogenic polydipsia, p-value $=0.3$ ), nor severity of polydipsia (moderate vs. severe polydipsia, $p$-value $=0.6$ ).

\section{Serum cortisol levels in response to ACTH stimulation}

Basal serum cortisol levels were similar for patients with primary polydipsia (median $445 \mathrm{nmol} / \mathrm{I}$ (IQR $395,551)$ ) as compared to healthy volunteers (median $530 \mathrm{nmol} / \mathrm{I}(\mathrm{IQR} 395,747)$, $\mathrm{p}$-value $=0.22)$. In response to ACTH stimulation, serum cortisol levels in patients with primary polydipsia increased to 618 $\mathrm{nmol} / \mathrm{I}(\mathrm{IQR} 580,684)$ and in healthy volunteers to $636 \mathrm{nmol} / \mathrm{I}(\mathrm{IQR} 512,910)(\mathrm{p}$-value $=0.77)$, respectively (Fig. 3). Estimated difference in patients with primary polydipsia compared to healthy volunteers was $89 \mathrm{nmol} / \mathrm{l}(95 \%-\mathrm{Cl}:-172,-7), \mathrm{p}$-value $=0.04)$, which was not significant after adjusting for sex and the intake of oral contraceptive pills (estimated difference $-34 \mathrm{nmol} / \mathrm{I}(95 \%-\mathrm{Cl}:-105,36)$, p-value $=0.4)$.

There was no difference in serum cortisol levels in response to ACTH stimulation for the subgroups type of polydipsia (psychogenic vs. habitual polydipsia, estimated difference - $21 \mathrm{nmol} / \mathrm{l}(95 \%-\mathrm{Cl}$ - $-103,61), \mathrm{p}$ value $=0.6$ ). Patients with severe polydipsia had lower serum cortisol levels in response to ACTH stimulation than patients with moderate polydipsia (estimated difference - $107 \mathrm{nmol} / \mathrm{l}(95 \%-\mathrm{Cl}:-185,-28)$, $\mathrm{p}$-value $=0.009)$. This remained statistically significant after adjusting for age, sex, and oral contraceptives ( $p$-value $<0.001)$. Further analysis revealed that basal serum cortisol levels (measured on the second study day, when patients had free access to water) were lower in patients with severe polydipsia as compared to moderate polydipsia (basal serum cortisol severe polydipsia $386 \mathrm{nmol} / \mathrm{l}$ vs. moderate polydipsia $537 \mathrm{nmol} / \mathrm{l}$, $\mathrm{p}$-value $=0.02$ ) while stimulated cortisol levels were not different (stimulated serum cortisol severe polydipsia $607 \mathrm{nmol} / \mathrm{l}$ vs. moderate polydipsia $670 \mathrm{nmol} / \mathrm{l}, \mathrm{p}$-value = $0.14)$. 


\section{Discussion}

This study shows no difference between the HPA axis activity in patients with primary polydipsia compared to healthy volunteers as indicated by comparable measurements of circadian plasma cortisol, 24-hour urinary cortisol levels, and cortisol levels in response to ACTH stimulation. The results do not support the hypothesis that increased fluid intake alters HPA axis activity in patients with primary polydipsia.

One of the theories around primary polydipsia is a downregulation of the HPA axis caused by constant suppression of vasopressin [17]. It has been shown that vasopressin, besides regulating water-sodium homeostasis, is a stress hormone that co-activates the pituitary to release ACTH [18]. Of note, vasopressin regulates the osmolality via magnocellular neurons and axonal transport to the posterior pituitary while it co-activates the anterior pituitary via parvocellular neurons through the portal system [19]. This pathophysiological difference of vasopressin release could explain that we observed no effect on stress-reactivity.

In patients who suffer both from schizophrenia and psychogenic polydipsia, Goldman et al. identified an overactivation of the HPA axis $[8,10,11,20]$. Main findings have been a non-suppression of cortisol in the dexamethasone suppression test and diminished glucocorticoid negative feedback upon ACTH stimulation [7, 9]. The observed difference in HPA axis activity of our findings compared to Goldman et al. is possibly explained by patient's selection: patients with schizophrenia may represent a distinct subpopulation with additional neuroendocrine alterations that could account for the altered HPA axis response. Also, the alteration of the HPA axis might be dependent on the presence and severity of water imbalance, i.e., hyponatremia, and acute psychotic exacerbation as often present in Goldman's studies. In our sample, most patients had habitual polydipsia and only one patient was suffering from schizophrenia (stable at the time of study participation), and except for two patients who experienced mild hyponatremia in the afternoon (data not shown), our patients had laboratory parameters within the normal range. We only found lower basal cortisol levels but an adequate response to ACTH in the subgroup of severe polydipsic patients on the second day when patients had free access to water but not after water restriction as outlined in the first measurement of the circadian cortisol profile. These results may point to a different stress reactivity according to the severity of water imbalance.

Our results indicate a significant difference in the circadian salivary but not serum cortisol levels between patients with primary polydipsia and healthy volunteers. Measuring salivary cortisol has been an easy to use and practical surrogate marker for free serum cortisol, making it possible to collect samples during daily routines with a high accuracy [21-23]. However, it has been noted that the method is prone to errors due to contamination of saliva and inadequate sample volumes [22-24]. Since the difference in circadian cortisol levels was present in salivary measurements only, we interpret the difference found between patients with primary polydipsia and healthy volunteers rather as a dilution effect due to the larger amount of water consumed by patients with primary polydipsia. This effect might even persist if patients abstain from water for several hours as the first saliva sample in the morning was taken after an 
overnight fasting period of at least 12 hours. In a small study in athletes, short term increased fluid intake reduced salivary cortisol levels [25] Based on these results, clinicians should inquire about the fluid intake of patients when sampling salivary cortisol, especially when faced with unexpectedly low cortisol levels.

Research has shown that short term increased fluid intake and consecutive polyuria led to elevated free urinary cortisol levels in healthy volunteers $[26,27]$. Our results show a similar 24-hour free urinary cortisol excretion in patients with primary polydipsia and healthy volunteers. Hence, we consider 24-hour free urinary cortisol levels to be a reliable measurement that can be used also in individuals with a high fluid intake. Furthermore, there was no correlation between amount of drinking nor urinary output and 24hour urinary free cortisol. An alternative explanation to the previous findings could be that healthy participants forced to an increased fluid intake may experience elevated stress levels and therefore increased 24-hour free urinary cortisol excretion.

The following limitations of our study should be considered: First, we did not measure serum cortisol after dexamethasone stimulation test. However, since our findings in the majority of did not indicate any difference between patients with primary polydipsia and healthy volunteers, we surmise that the results from a dexamethasone stimulation test would have been no different. Second, our assessment of the circadian cortisol might not have detected subtle changes given that the frequency of sampling was limited to three serum and five salivary measurements per 24 hours, respectively. As we however have similar results for the collected timepoints, we consider our results reliable. Third, even though this is so far the largest study investigating the HPA axis in patients with primary polydipsia, the number of participants was still limited to 34 subjects. This led to small subgroups, i.e., type and severity of polydipsia. However, it is the largest sample outside the psychiatric setting and thus adds valuable information on the pathophysiology of primary polydipsia.

In summary, our data show that there is no difference between the HPA axis activity in patients with primary polydipsia and healthy volunteers assessed by different cortisol measurements. These data do not support the hypothesis that increased chronic daily fluid intake leads to an altered HPA axis.

Moreover, our results suggest using salivary cortisol with caution in patients with increased fluid intake as a dilution effect might lead to false-low levels. In contrast, 24-hour free urinary cortisol excretion seems to be reliable even in individuals with increased fluid intake.

\section{Declarations}

\section{Acknowledgement}

We thank our participants for taking part in our study. In addition, we thank the support study staff and laboratory personnel at the University Hospital of Basel, especially Nina Hutter, Cemile Bathelt, Joyce Santos de Jesus, Karin Wild, Céline Bürgi, Silke Purschke, Vanessa Grassedonio, and Klaus Ehrlich for their most helpful support during the study.

\section{Statement of Ethics}


The studies were preregistered on ClinicalTrials.gov (NCT03141632, NCT02770885) and approved by the Ethical Committee of Northwest and Central Switzerland. Written informed consent was obtained from each participant after full explanation of the purpose and nature of all procedures used.

\section{Conflict of Interest Statement}

The authors declare no conflict of interest.

\section{Funding Sources}

The study was investigator initiated and was supported by a grant from the Swiss National Foundation to M. Christ-Crain (SNF-162608), and from a grant the University Hospital Basel, Switzerland, to B. Winzeler. C. O. Sailer was supported by a Young Talents in Clinical Research grant of the Swiss Academy of Medical Sciences and of the G.\& J. Bangerter-Rhyner Foundation and received a top-up stipend from the PhD Program Health Sciences, Basel University, Switzerland.

\section{Author Contributions}

C.O.S., J.R., M.C.-C., and B.W. contributed to the study design. C.O.S., I.d.C., and B.W. collected the data. C.O.S. and J.M.K. performed the statistical analysis. C.O.S. and J.M.K. drafted the manuscript and all authors provided intellectual input and critically reviewed the manuscript. M.C.-C. and B.W. supervised the work. All authors approved the final version of the manuscript and agree with its submission.

\section{References}

1. E. Mercier-Guidez, G. Loas, Eur. Psychiatry 15, 306 (2000)

2. G.L. Robertson, Best Pract. Res. Clin. Endocrinol. Metab. 30, 205 (2016)

3. C.O. Sailer, W. Bettina, C. Mirjam, Swiss Med. Wkly. 147, 1 (2017)

4. B. Dundas, M. Harris, M. Narasimhan, Curr. Psychiatry Rep. 9, 236 (2007)

5. J. de Leon, C. Verghese, J.I. Tracy, R.C. Josiassen, G.M. Simpson, Biol. Psychiatry 35, 408 (1994)

6. M.B. Goldman, G.L. Robertson, D.J. Luchins, D. Hedeker, G.N. Pandey, Arch. Gen. Psychiatry 54, 443 (1997)

7. M.B. Goldman, G. Wood, M.B. Goldman, M. Gavin, S. Paul, S. Zaheer, G. Fayyaz, R.S. Pilla, J. Clin. Endocrinol. Metab. 92, 698 (2007)

8. M.B. Goldman, I.J. Torres, S. Keedy, M. Marlow-O'Connor, B. Beenken, R. Pilla, Hippocampus 17, 554 (2007)

9. M.B. Goldman, L. Blake, R.C. Marks, D. Hedeker, D.J. Luchins, Am. J. Psychiatry 150, 653 (1993)

10. M.B. Goldman, L. Wang, C. Wachi, S. Daudi, J. Csernansky, M. Marlow-O'Connor, S. Keedy, I. Torres, Behav. Brain Res. 218, 106 (2011) 
11. M.B. Goldman, Schizophr Res. 157, 204 (2014)

12. W. Fenske, J. Refardt, I. Chifu, I. Schnyder, B. Winzeler, J. Drummond, A. Ribeiro-Oliveira, T. Drescher, S. Bilz, D.R. Vogt, U. Malzahn, M. Kroiss, E. Christ, C. Henzen, S. Fischli, A. Tönjes, B. Mueller, J. Schopohl, J. Flitsch, G. Brabant, M. Fassnacht, M. Christ-Crain, N Engl. J. Med. 379, 428 (2018)

13. B. Winzeler, N. Cesana-Nigro, J. Refardt, D.R. Vogt, C. Imber, B. Morin, M. Popovic, M. Steinmetz, C.O. Sailer, G. Szinnai, I. Chifu, M. Fassnacht, and M. Christ-Crain, Lancet 394, 587 (2019)

14. B. Winzeler, C. Sailer, D. Coynel, D. Zanchi, D. Vogt, S. Urwyler, J. Refardt, M. Christ-Crain, J. Clin. Invest. (2021)

15. B. Winzeler, I. Conceição, J. Refardt, C.O. Sailer, G. Dutilh, and M. Christ-Crain, Endocrine 1 (2020)

16. T.R. Core, (2018)

17. D.J. Luchins, K.W. Nettles, M.B. Goldman, Biol. Psychiatry 42, 767 (1997)

18. M. Katan, N. Morgenthaler, I. Widmer, J.J. Puder, C. Konig, B. Muller, and M. Christ-Crain, Neuro Endocrinol Lett 29, 341 (2008)

19. G. Aguilera, C. Rabadan-Diehl, Regul. Pept. 96, 23 (2000)

20. D.J. Luchins, Biol. Psychiatry 28, 87 (1990)

21. C. Kirschbaum, D.H. Hellhammer, Neuropsychobiology 22, 150 (1989)

22. J. Blair, J. Adaway, B. Keevil, R. Ross, Curr. Opin. Endocrinol. Diabetes Obes. 24, 161 (2017)

23. C. Duplessis, D. Rascona, M. Cullum, E. Yeung, Mil Med. 175, 340 (2010)

24. A. Clow, N. Smyth, Int. Rev. Neurobiol (Academic Press Inc., 2020), pp. 1-16

25. T. Backes, P. Horvath, K. Kazial, Biol. Sport 32, 275 (2015)

26. M.V. Mericq, G.B. Cutler, J. Clin. Endocrinol. Metab. 83, 682 (1998)

27. M. Fenske, Steroids 71, 1014 (2006)

\section{Figures}



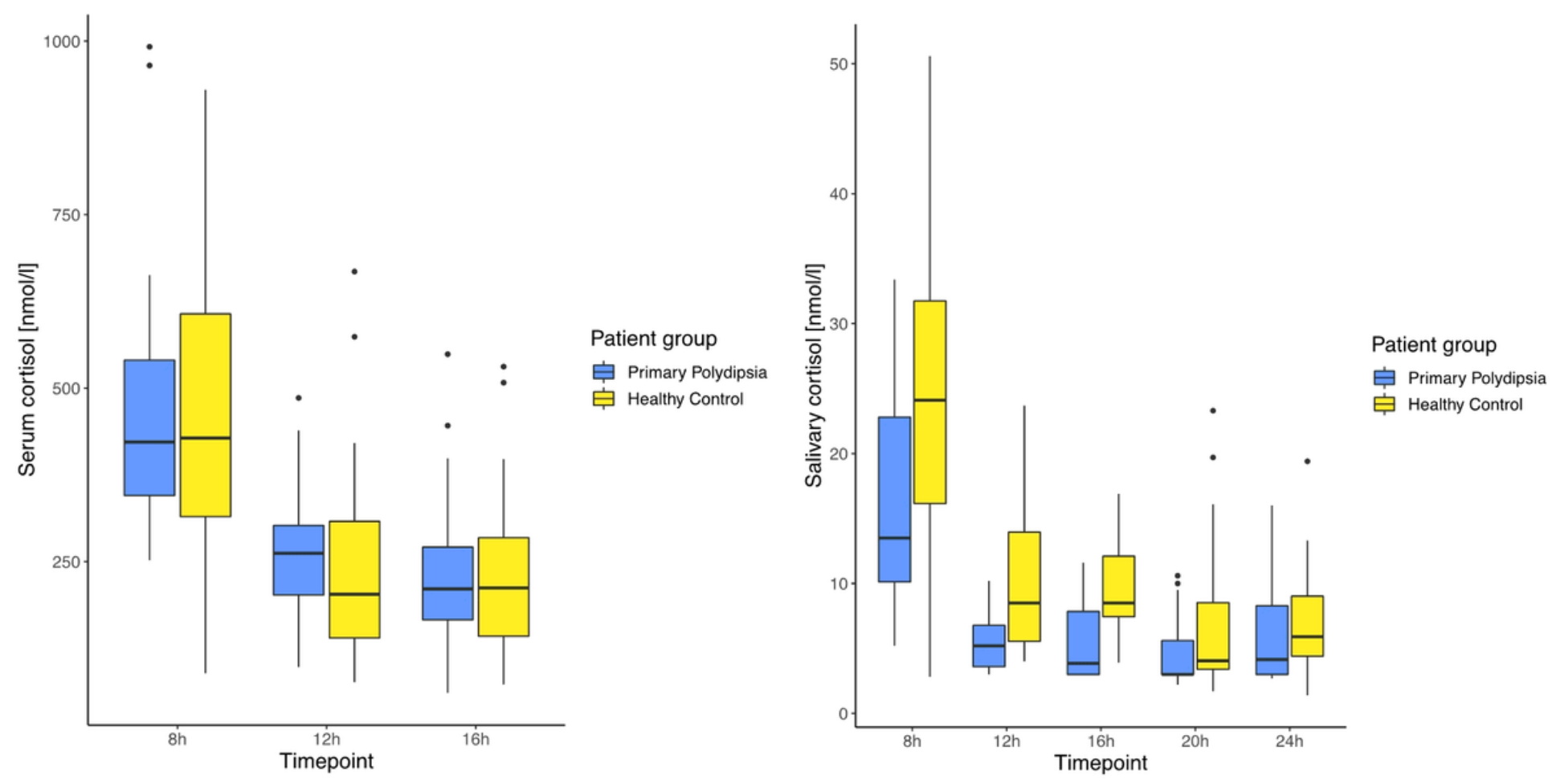

Figure 1

Differences in cortisol values between patients with primary polydipsia and healthy volunteers. (a) Circadian serum cortisol levels in patients with primary polydipsia vs. healthy controls. Thick line indicates the median; box indicates the IQR; whiskers include all points within the range of $1.5 \times$ the IQR. (b) Circadian salivary cortisol levels in patients with primary polydipsia vs. healthy controls. Thick line indicates the median; box indicates the IQR; whiskers include all points within the range of 1.5x the IQR. 


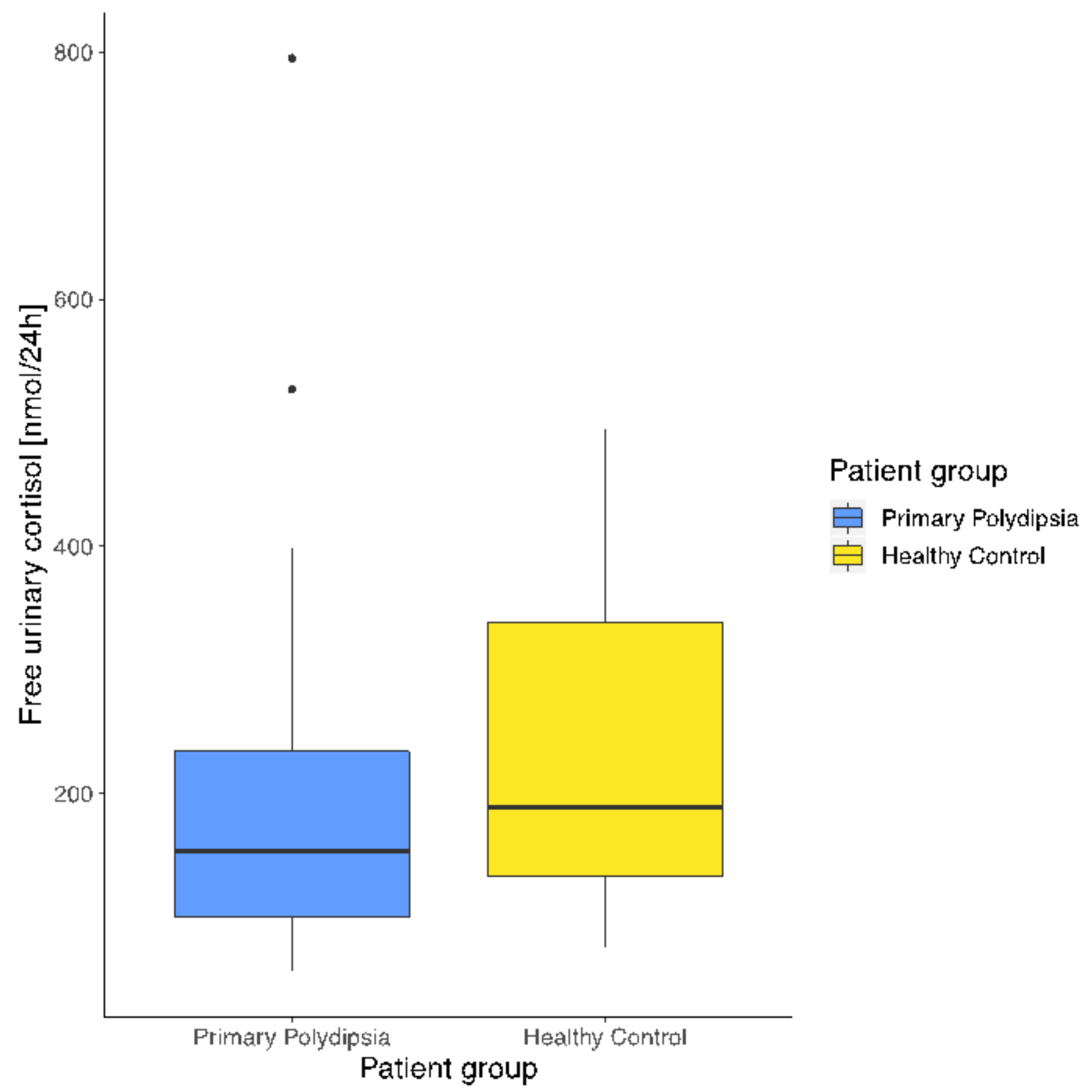

\section{Figure 2}

24-hour urinary free cortisol in patients with primary polydipsia vs. healthy volunteers. Thick line indicates the median; box indicates the IQR; whiskers include all points within the range of $1.5 \times$ the IQR. 


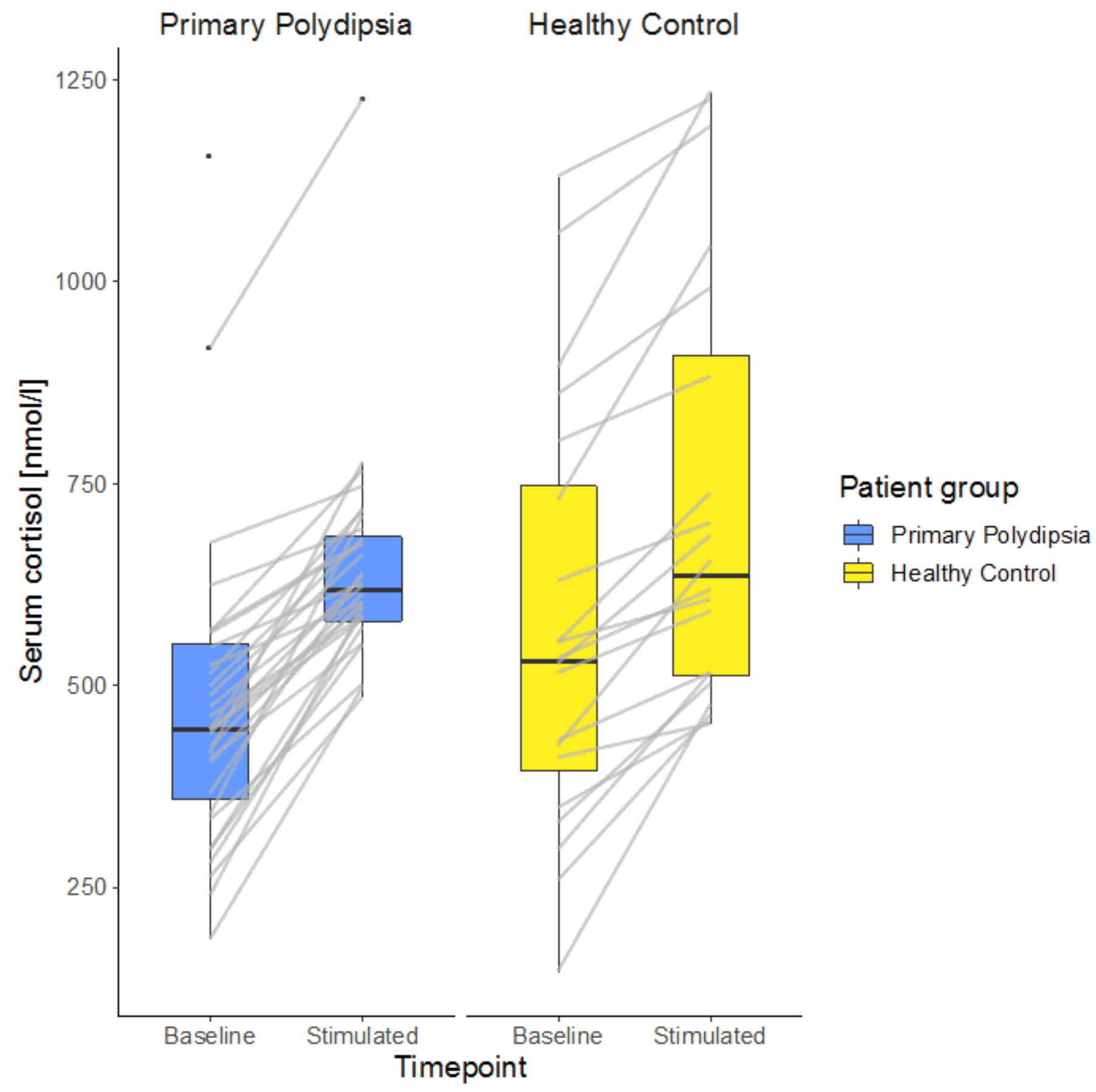

\section{Figure 3}

Serum cortisol upon ACTH stimulation for patients with primary polydipsia and healthy controls. Thick line indicates the median; box indicates the IQR; whiskers include all points within the range of $1.5 \times$ the IQR. Grey lines indicate the change in serum cortisol levels per individual person. 